\title{
Unique Temperature Dependence of Selectively Liquid-crystal-filled Photonic Crystal Fibers
}

\author{
Bing Sun, Zuxing Zhang, Wei Wei, Chao Wang, Changrui Liao, Lin Zhang, and Yiping Wang, Fellow, \\ IEEE
}

\begin{abstract}
We demonstrate a unique temperature-dependent characteristic of the selectively liquid-crystal-filled photonic crystal fiber (SLCPCF), which is realized by selectively infiltrating liquid crystal into a single air hole located at the second ring near the core of the PCF. Three-resonance dips are observed in the transmission spectrum. Theoretical and experimental investigations reveal that the three-resonance dips all result from the coupling between the $\mathrm{LP}_{01}$ core mode and the rod modes, i.e., $L P_{03}$ and $L_{P_{51}}$. Then, we find that the dips shift induced by temperature shows good agreements with the thermo-optic performance of the LC employed in the Ref. [1]. Furthermore, the dips shift greatly with changes in temperature, providing a method to achieve temperature measurement in such a compact structure.
\end{abstract}

Index Terms-Photonic crystal fibers; Liquid-crystal devices; Fiber optics sensors; Fiber optics

\section{INTRODUCTION}

$\mathrm{I}$ nfiltration of liquid crystals (LCs) into the air holes of 1 photonic crystal fibers (PCFs) has been becoming a good infusion candidate for realizing thermally tunable devices. On one hand, the guiding mechanism of PCF from modified total internal reflection to photonic bandgap (PBG) guidance could be implemented by filling all of PCF's holes with LCs [2-11]. In special, Larsen et al. [2] proposed a thermo-optic switching based on that the PBG transmission could be totally depressed around the LC's clearing point temperature. Filling of the complete cladding region and coupling processes of waveguides were investigated by Lorenz et al. [3-4, 6, 8] and Wahle et al. [9-10]. Recently, we demonstrated a liquid-crystal-filled PCF and investigated its temperature response nearby a unique LC's clearing point [11].

On the other hand, selective infiltration techniques enabled a controllable and repeatable fabrication of tunable PCF devices

This work was supported by National Natural Science Foundation of China $(61505119,11174064,61308027$, and 61377090) and Brain Gain Foundation of Nanjing University of Posts and Telecommunications (NY215040). Corresponding author: Yiping Wang (e-mail: ypwang@szu.edu.cn).

Bing Sun, Zuxing Zhang, Wei Wei are with the School of Optoelectronic Engineering, Nanjing University of Posts and Telecommunications, Nanjing 210023, China (e-mail: b.sun@njupt.edu.cn; zxzhang@ njupt.edu.cn; weiwei@njupt.edu.cn).

Chao Wang, Changrui Liao, Yiping Wang are with the Key Laboratory of Optoelectronic Devices and Systems of Ministry of Education and Guangdong Province, College of Optoelectronic Engineering, Shenzhen University, Shenzhen 518060, China (e-mail: 3752601@qq.com; cliao@szu.edu.cn).

Lin Zhang is with the Aston Institute of Photonic Technologies, Aston University, Birmingham B4 7ET, UK (e-mail: 1.zhang@aston.ac.uk).
[12-14]. Nevertheless, few reports investigated its temperature properties by taking advantage of selectively filling LC into PCFs except for the previous reports [15-17]. However, the heating range was limited and its temperature-dependent properties were not implemented completely.

In this letter, we experimentally investigate the temperature responses of selectively liquid-crystal-filled photonic crystal fibers (SLCPCFs) ranging from $-45^{\circ} \mathrm{C}$ to $110{ }^{\circ} \mathrm{C}$ in detail. We observe three-resonance dips in the measured transmission spectrum and theoretically conclude that these resonance dips result from the coupling between the $\mathrm{LP}_{01}$ core mode and the rod modes, i.e., $\mathrm{LP}_{03}$ and $\mathrm{LP}_{51}$, respectively. Interesting is that the LC possesses special phase transition property (from anisotropy to isotropy) at $58{ }^{\circ} \mathrm{C}$. Furthermore, the SLCPCF demonstrates a method to achieve high-sensitivity temperature sensing (with a sensitivity of $40 \mathrm{~nm} /{ }^{\circ} \mathrm{C}$ ) in such a compact structure.

\section{PROPERTIES OF LIQUid CRYSTAL AND DEVICE FABRICATION}

A commonly used nematic liquid crystal (NLC) from Merck, i.e., E7 has been employed. Anisotropic optical properties of E7 can be described by the extraordinary and ordinary refractive index of $n_{e}$ and $n_{o}$ when the temperature is lower than a clearing point temperature, $T_{N I}$, of $58{ }^{\circ} \mathrm{C}$. However, $n_{o}$ takes dominating effect in modifying the wave-guiding properties of the PCF according to the reference [18]. Firstly, $n_{o}$ decreases with the rise in temperature until $n_{o}$ tend to be a stable value. Then, $n_{o}$ increases further with the rise in temperature. Then, $n_{e}$ equals to $n_{o}$ and the anisotropy vanishes when the temperature is higher than $T_{N I}[1]$.

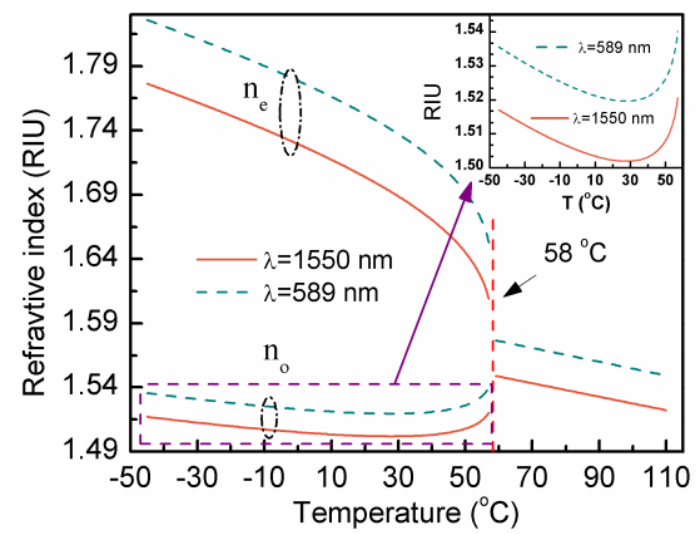

Fig. 1. Temperature-dependent refractive indices of E7 at $\lambda=589 \mathrm{~nm}$ and $\lambda=1550 \mathrm{~nm}$, respectively. The inset shows the enlarged temperature characteristics of the refractive indices of LC, i.e., E7. 
A schematic flowchart of the selective infiltration process is illustrated in Fig. 2. Firstly, an optimized splicing parameters (prefusion power, standard -25 bit; prefusion time, $180 \mathrm{~ms}$; overlap, $6 \mu \mathrm{m}$; fusion power one, standard-30 bit; fusion time one, $300 \mathrm{~ms}$; offset, L-30 $\mu \mathrm{m}$ ) are involved to avoid collapsing air holes in the cladding. Secondly, a section of $\sim 10-\mu \mathrm{m}$-long SMF away from the splicing point on the fiber surface has been held back by means of a precision cleaving device. As a result, the spliced end of the PCF is fully blocked by the $\sim 10-\mu \mathrm{m}$-long SMF. Furthermore, with this thickness of the SMF, all air holes in the cladding can be clearly observed with the microscopic objectives employed, as shown in Fig. 2(b). Thirdly, the fs laser pulses $(\lambda=800 \mathrm{~nm})$ of 120 -fs duration with repetition rate of 1 $\mathrm{kHz}$ are focused onto the fiber samples by microscopic objectives. Moreover, the on-target pulse energy could be precisely controlled by rotating the half-wave plate incorporated with a polarizer. Fiber samples are mounted onto a computer controlled three-dimensional translation stage, as shown in Fig. 2(c). Intuitively, the microscopic images corresponding to Fig. 2(a), (b), and (c) are depicted in the lower right side of Fig. 2. In this experiment, the air hole on one end of a 1-m-long PCF is selectively opened and high pressure gas is applied to the one selected air-columns of the PCF. In the meantime, the other end of the $\sim 1$-m-long PCF is placed in the splicer. Thus, the whole endface collapsed except the selected air hole corresponding to the other end is opened.

Next, one end of the selectively opened $\sim 1$-m-long PCF is sealed in a pressure tube connected to an injection syringe, and the other end of the PCF is immerged in a vessel holding for E7. The injection syringe is fixed on an electrically controlled moving stage to provide a constant pressure difference. When pulling the plunger of the injection syringe, it produces a vacuum pressure in the tube and in the single channel of the PCF, which is thereby filled with LC, selectively.

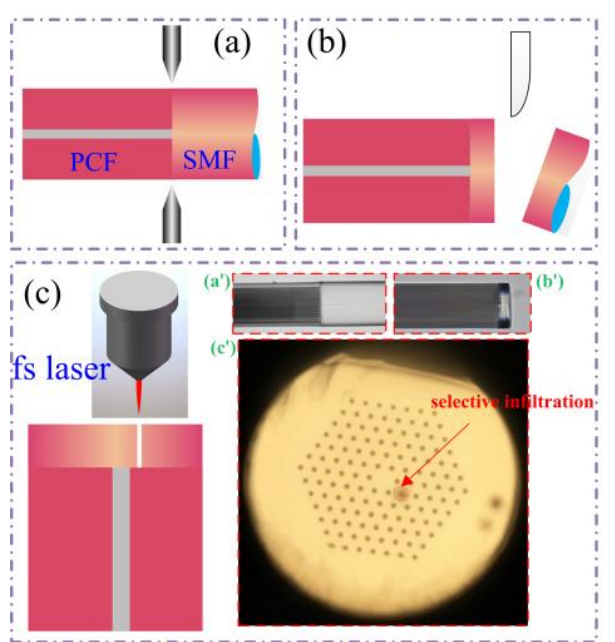

Fig. 2. Flowchart of selective infiltration assisted with femtosecond laser micromachining, (a) fusion splicing; (b) precision cleaving; (c) laser drilling.

\section{EXPERIMENTAL RESULT AND DisCUSSION}

We employ a large mode area PCF (ESM-12), as shown in Fig. 3(a), the air holes of the PCF with a core diameter of $9 \mu \mathrm{m}$ have an average diameter of $3.6 \mu \mathrm{m}$ and are arranged in a hexagonal pattern with an average pitch of $7.9 \mu \mathrm{m}$. The liquid crystal, i.e., E7 employed in this experiment is stored in a vessel, as shown in Fig. 3(b). Considering the material dispersion, we numerically calculate the effective refraction index of the core mode, i.e., $\mathrm{LP}_{01}$ and the satellite rod modes, i.e., $\mathrm{LP}_{03}$ and $\mathrm{LP}_{51}$, respectively, at $25{ }^{\circ} \mathrm{C}$, by using the full-vector finite-element method. As shown in Fig. 3(c), at 25 ${ }^{\circ} \mathrm{C}$, the dispersion curve of the core $\mathrm{LP}_{01}$ mode (the black solid curve) intersects that of the $\mathrm{LP}_{51}$ mode of rod (the red solid curve) near $1400 \mathrm{~nm}$. Meanwhile, it can intersect the dispersion curves of the $\mathrm{LP}_{03}$ mode of rod (the magenta dashed curves) near $1220 \mathrm{~nm}$ and $1250 \mathrm{~nm}$, so there will be three resonance dips. The insets are the simulated mode profiles of the $\mathrm{LP}_{01}$ mode and the satellite rod modes $\left(\mathrm{LP}_{03}\right.$ and $\left.\mathrm{LP}_{51}\right)$. Note that the other rod modes are not plotted in Fig. 3(c) as their dispersion curves cannot intersect with the core $\mathrm{LP}_{01}$ curve in the spectral range from 1100 to $1700 \mathrm{~nm}$. The transmission spectrum of the SLCPCF at $25{ }^{\circ} \mathrm{C}$ is shown in Fig. 3(c). The measured dip positions agree well with the simulated results except that the slight difference between the measured and the calculated dip position near $1400 \mathrm{~nm}$. We attribute the slight difference to that the actual RI values and the geometrical structural parameters of the fiber cannot be fully consistent with the values used in the theoretical calculations. In the three resonance areas we select the resonant peak near $1400 \mathrm{~nm}$ as characteristic peaks, and mark it as $\lambda_{\text {res } 1}$.

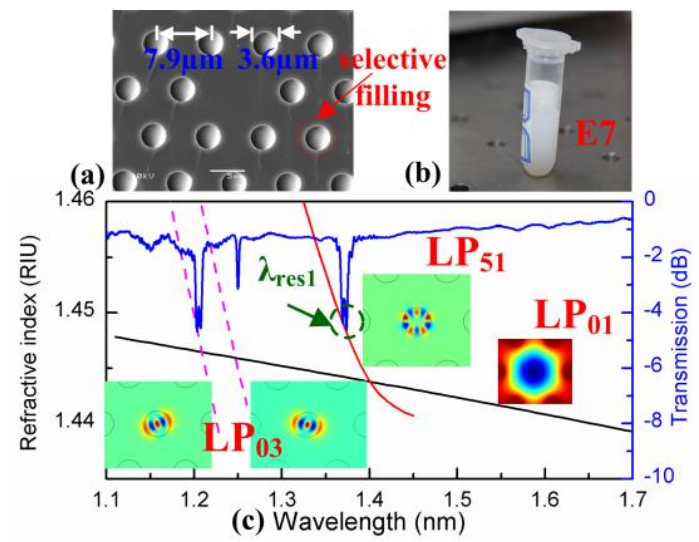

Fig. 3. Cross-sectional SEM images of the PCF employed (a); the liquid crystal employed, i.e., E7 (b); Dispersion curves of the core mode $\mathrm{LP}_{01}$ and the satellite rod modes $\left(\mathrm{LP}_{03}, \mathrm{LP}_{51}\right)$ at $25{ }^{\circ} \mathrm{C}$; the blue solid line represents the transmission spectrum of liquid crystal selective filled PCF (c). The insets are the simulated mode profiles of the core mode $\mathrm{LP}_{01}$ and the satellite rod modes $\left(\mathrm{LP}_{03}, \mathrm{LP}_{51}\right)$.

The infiltrated PCF sample is then cut back for a small distance and spliced with SMF such that a number of different infiltrated PCF lengths are achieved. For different infiltration length $L$, the transmission spectra of the sample at room temperature are measured and shown in Fig. 4, respectively. It can be seen that the transmission spectra with different $L$ present the same resonant dip positions near 1220, 1250, and $1400 \mathrm{~nm}$, respectively, except that the depth of the dips are deeper with the increasing $L$. This reference [19] explained well that it is important to match the infiltration length $L$ as closely as possible to $L_{c}$ to optimize the depths of the resonant dip, and then to optimize the detection limit of the device, where $L_{c}$ represents the coupling length. More significantly, the fusion splicing between the infiltrated PCF and the SMF are optimized 
with an insertion loss of less than $2 \mathrm{~dB}$ via the improved splicing parameters.

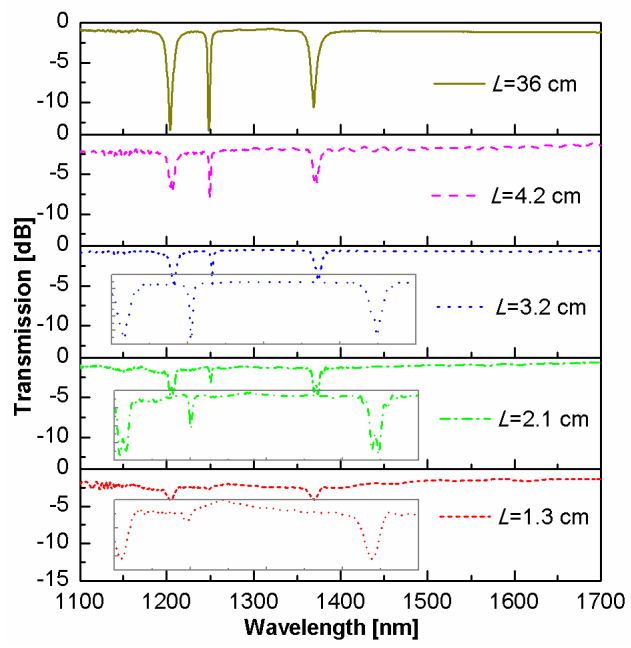

Fig. 4. The measured transmission spectra of the samples at room temperature for different infiltration length $L$.

The SLCPCF with 2.1-cm length is placed in a column oven to investigate its transmission spectra at different temperature with a supercontinuum source and an optical spectrum analyzer. In addition, a differential thermocouple is used to measure the temperature with an accuracy of $0.1^{\circ} \mathrm{C}$. Figure $5(\mathrm{a})$ shows the transmission spectra of the SLCPCF, corresponding to the temperature varying from $-45^{\circ} \mathrm{C}$ to $30{ }^{\circ} \mathrm{C}$. The resonant coupling firstly appears in a wide spectral range near $1350 \mathrm{~nm}$ at $30{ }^{\circ} \mathrm{C}$ shown as the pink line. Then another resonant dips disappear with the increasing temperature and the corresponding dips depth increase gradually. By tracing $\lambda_{\text {res } 1}$, the temperature sensitivity can be obtained, as shown in Fig. 5(b). The curve is fit by an exponential function approximation with a high goodness-of-fit coefficient $\mathrm{R}^{2}$ value. We can see that the rise in temperature results in a 'blue' shift of $\lambda_{\text {res } 1 \text {. }}$
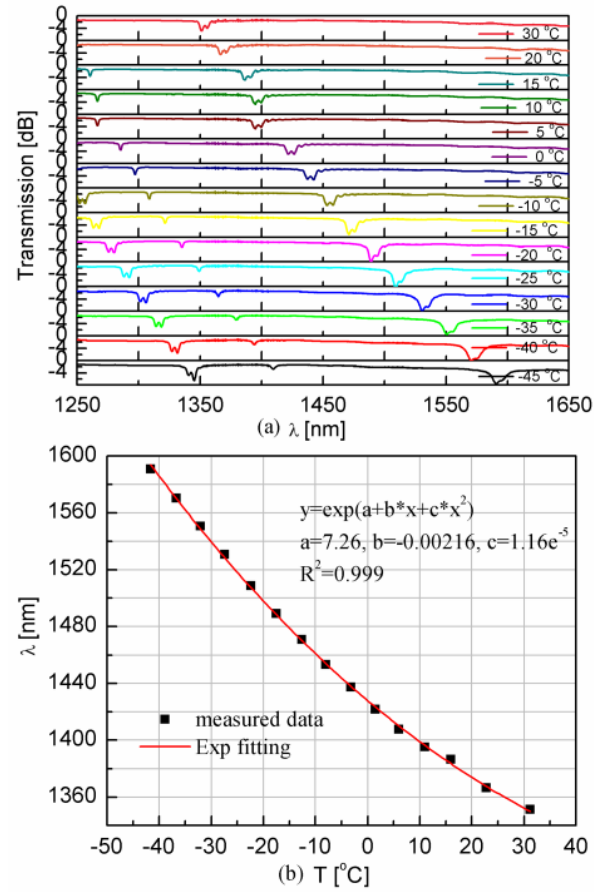

Fig. 5. (a) The transmission spectra of the SLCPCF corresponding to variations in temperature from $-45^{\circ} \mathrm{C}$ to $30{ }^{\circ} \mathrm{C}$; (b) Temperature sensitivity of $\lambda_{\text {res } 1}$.

The further rise in temperature, from $40{ }^{\circ} \mathrm{C}$ to $57^{\circ} \mathrm{C}$, results in a 'red' shift of $\lambda_{\text {res } 1}$ and the shift can be fit by an allometric-2 function approximation with a coefficient $R^{2}$ value $\left(R^{2}=0.995\right)$. In special, as the temperature approaches the clearing point of $58{ }^{\circ} \mathrm{C}$, a very slight temperature variation result in an evident shift, as shown in Fig. 6. For instance, a wavelength shift as high as $12.06 \mathrm{~nm}$ is obtained as the temperature, i.e., T, varying from $57.0{ }^{\circ} \mathrm{C}$ to $57.3{ }^{\circ} \mathrm{C}$. Therefore, experimental sensitivity exceeding $40.2 \mathrm{~nm} /{ }^{\circ} \mathrm{C}$ is achieved. The highest sensitivity for temperature is up to $40.2 \mathrm{~nm} /{ }^{\circ} \mathrm{C}$ at $57.3{ }^{\circ} \mathrm{C}$, which is at least 10 times larger than the literature reported in [15-16], 3 times larger than that reported in [20-21] as well. More interesting, the observed trend of $\lambda_{\text {res } 1}$ is different with that was previously reported [22-23].
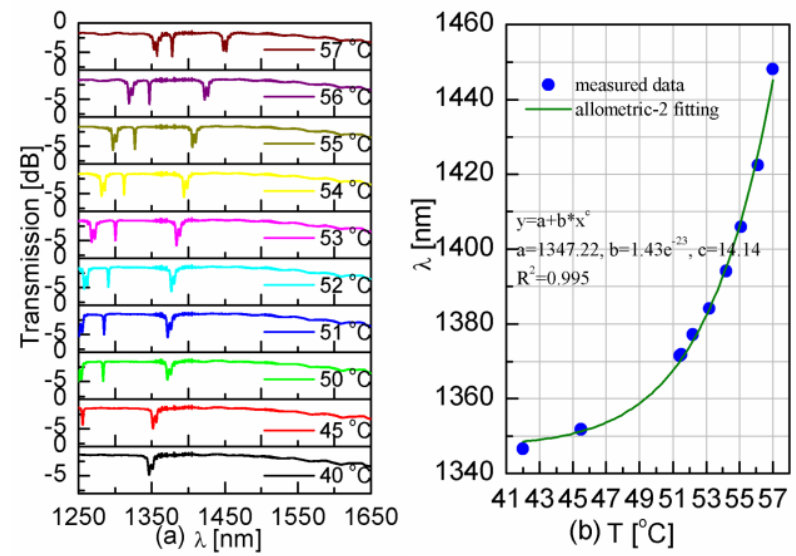

Fig. 6. (a) The transmission spectra of the SLCPCF corresponding to variations in temperature from $40{ }^{\circ} \mathrm{C}$ to $57^{\circ} \mathrm{C}$; (b) Temperature sensitivity of $\lambda_{\text {ress }}$.

When the temperature is higher than $T_{N I}$, the anisotropy of E7 vanishes. Then, the refractive index in the isotropic phase decreases linearly as the temperature increases, showing similar thermo-optic coefficient with refractive index matched liquid from Cargille Labs [24]. We may deduce that the two resonant dips $\left(\lambda_{\text {res } 2}\right.$ and $\left.\lambda_{\text {res } 3}\right)$ shown in the measured transmission result from the coupling between the fundamental mode $\left(\mathrm{HE}_{11 y}\right.$ and $\left.\mathrm{HE}_{11 x}\right)$ and the higher order modes $\left(\mathrm{HE}_{21 y}, \mathrm{TM}_{1}\right.$ and $\left.\mathrm{HE}_{21 x}, \mathrm{TE}_{1}\right)$ in Fig. 7. Linear fit method is applied to the linear region of the curve and a sensitivity of $\sim-3.7 \mathrm{~nm} /{ }^{\circ} \mathrm{C}$ is achieved.
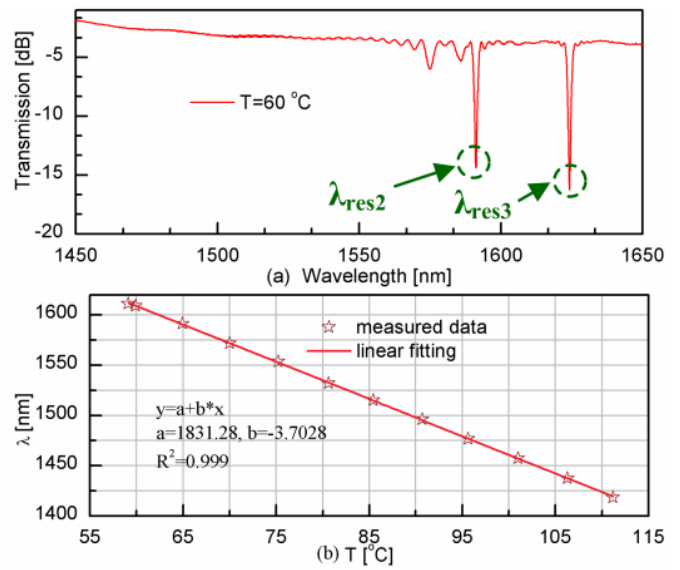

Fig.7. (a) The transmission spectra of the SLCPCF corresponding to variations in temperature from $59{ }^{\circ} \mathrm{C}$ to $110{ }^{\circ} \mathrm{C}$; (b) Temperature sensitivity of $\lambda_{\text {res } 2}$. 
Figure 8 shows that the dip shifts towards shorter wavelength, then towards longer wavelength. Moreover, the LC phase has changed from anisotropic to isotropic in the temperature of $58{ }^{\circ} \mathrm{C}$. Close to the temperature of $58^{\circ} \mathrm{C}$, the dip shifts drastically with high sensitivity. In the isotropic phase, the LC presents similar optical characteristics with refractive index matched liquids from Cargille Labs. Interestingly, the transformation between anisotropic phase and isotropic phase is reversible. The good agreement between theory and experiment confirms that the structure observed does give the expected optical response.

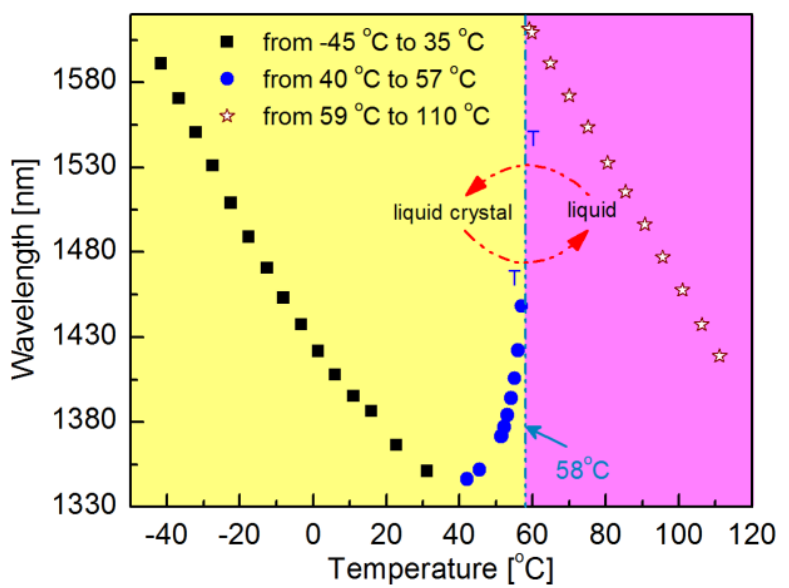

Fig. 8. The transmission spectra of the SLCPCF corresponding to variations in temperature from $-45^{\circ} \mathrm{C}$ to $110{ }^{\circ} \mathrm{C}$.

\section{CONCLUSION}

In conclusion, we investigated the temperature tuning properties of the SLCPCFs from $-45^{\circ} \mathrm{C}$ to $110^{\circ} \mathrm{C}$. Because of the special temperature responses of LC's indices and its phase transition property, the SLCPCF transmission was found to have different temperature responses at different temperature ranges. In special, we experimentally observed that the SLCPCF transmissions follows different trends within the LC phase, where the shift of the resonant wavelengths can be described with a quadratic function, and the isotropic phase, where the shift of the resonant wavelengths can be described with a linear function. The temperature sensitivity of the reported fiber (with a selectively filled channel) was 3-10 times higher than in fibers previously studied [15-16, 20-21].

\section{REFERENCES}

[1] J. Li, S.-T. Wu, S. Brugioni, R. Meucci, and S. Faetti, "Infrared refractive indices of liquid crystals," J. Appl. Phys., vol. 97(073501), pp. 1-5, Mar. $18,2005$.

[2] T. T. Larsen, J. Broeng, D. S. Hermann, and A. Bjarklev, "Thermo-optic switching in liquid crystal infiltrated photonic bandgap fibres," Electronics Letters, vol. 39, no. 24, pp. 1719-1720, Nov. 27, 2003.

[3] A. Lorenz, R. Schuhmann, and H.-S. Kitzerow, "Switchable waveguiding in two liquid crystal-filled photonic crystal fibers," Appl. Opt., vol. 49, no. 24, pp. 3846-3853, Jul. 1, 2010.

[4] A. Lorenz, and H.-S. Kitzerow, "Efficient electro-optic switching in a photonic liquid crystal fiber," Appl. Phys. Lett., vol. 98, no. 24, pp. 241106, Nov. 13, 2011.

[5] T. T. Alkeskjold, J. Laegsgaard, A. Bjarklev, D. S. Hermann, J. Broeng, J. $\mathrm{Li}, \mathrm{S}$. Gauza, and S. T. Wu, "Highly tunable large-core single-mode liquid-crystal photonic bandgap fiber," Appl. Opt., vol. 45, no. 10, pp. 2261-2264, Apr. 1, 2006.
[6] A. Lorenz, H.-S. Kitzerow, A. Schwuchow, J. Kobelke, H. Bartelt, "Photonic crystal fiber with a dual-frequency addressable liquid crystal: behavior in the visible wavelength range," Opt. Express, vol. 16, no. 23, pp. 19375-19381, Nov. 7, 2008.

[7] J. Du, Y. Liu, Z. Wang, B. Zou, B. Liu, and X. Dong, "Liquid crystal photonic bandgap fiber: different bandgap transmissions at different temperature ranges," Appl. Opt., vol. 47, no. 29, pp. 5321-5324, Oct. 10, 2008.

[8] A. Lorenz, R. Schuhmann, H.-S. Kitzerow, "Infiltrated photonic crystal fiber: experiments and liquid crystal scattering model," Opt. Express, vol. 18, no. 4, pp. 3519-3530, Feb. 3, 2010.

[9] M. Wahle and H.-S. Kitzerow, "Measurement of group velocity dispersion in a solid-core photonic crystal fiber filled with a nematic liquid crystal," Opt. Lett., vol. 39, no. 16, pp. 4816-4819, Aug. 11, 2014.

[10] M. Wahle and H.-S. Kitzerow, "Electrically tunable zero dispersion wavelengths in photonic crystal fibers filled with a dual frequency addressable liquid crystal," Appl. Phys. Lett., vol. 107, no. 20, pp. 201114 , Nov. 16, 2015.

[11] B. Sun, Y. Huang, D. Luo, C. Wang, J. He, C. Liao, G. Yin, J. Zhou, S. Liu, J. Zhao, and Y.Wang, "Broadband Thermo-Optic Switching Effect Based on Liquid Crystal Infiltrated Photonic Crystal Fibers," IEEE Photonics J., vol. 7, no. 4, pp. 6802207-1, Aug. 1, 2015.

[12] Y. Wang, C. R. Liao, and D. N. Wang, "Femtosecond laser-assisted selective infiltration of microstructured optical fibers," Opt. Express, vol. 18, no. 17, pp. 18056-18060, Aug. 16, 2010.

[13] F. Wang, W. Yuan, O. Hansen, and O. Bang, "Selective filling of photonic crystal fibers using focused ion beam milled microchannels," Opt. Express, vol. 19, no. 18, pp. 17585-17590, Aug. 29, 2011.

[14] Y. Cui, P. Shum, D. J. J. Hu, G. Wang, G. Humber, and Q. Dinh, B, "Temperature sensor by using selectively-filled photonic crystal fiber Sagnac interferometer," IEEE Photon. J., vol. 4, no. 5, pp. 1801-1808, Oct. 2012.

[15] D. Hu, J. Lim, Y. Cui, K. Milenko, Y. Wang, P. Shum, and T. Wolinski, "Fabrication and characterization of a highly temperature sensitive device based on nematic liquid crystal-filled photonic crystal fiber," IEEE Photonics J., vol. 4, no. 5, pp. 1248-1255, Oct, 2012.

[16] D. Hu, P. Shum, J. Lim, Y. Cui, K. Milenko, Y. Wang, and T. Wolinski, "A compact and temperature-sensitive directional coupler based on photonic crystal fiber filled with liquid crystal 6CHBT," IEEE Photonics J., vol. 4, no. 5, pp. 2010-2016, Oct. 2012.

[17] M. Wahle, and H.-S. Kitzerow, "Liquid crystal assisted optical fibres," Opt. Express, vol. 22, no. 1, pp. 262-273, Jan. 2, 2014.

[18] T. T. Alkeskjold, J. Laegsgaard, A. Bjarklev, D. S. Hermann, Anawati, J. Broeng, J. Li, and S. T. Wu, "All-optical modulation in dye-doped nematic liquid crystal photonic bandgap fibers," Opt. Express, vol. 12, no. 24, pp. 5857-5871, Nov. 29, 2004.

[19] Darran K. C. Wu, Kwang Jo Lee, Vincent Pureur, and Boris T. Kuhlmey, "Performance of refractive index sensors based on directional couplers in photonic crystal fibers," J. Lightwave Technol., vol. 31, no. 22, pp. 3500-3510, Nov. 15, 2013.

[20] M. Yang and D. N. Wang, "Photonic crystal fiber With two infiltrated air holes for temperature sensing with excellent temporal stability," $J$. Lightwave Technol., vol. 30, no. 21, pp. 3407-3412, Nov. 1, 2012.

[21] D. K. C. Wu, B. T. Kuhlmey, and B. J. Eggleton, "Ultrasensitive photonic crystal fiber refractive index sensor," Opt. Lett., vol. 34, no. 3, pp. 322-324, Feb. 1, 2009.

[22] Y. Wang, M. Yang, D. N. Wang, and C. R. Liao, "Selectively infiltrated photonic crystal fiber with ultrahigh temperature sensitivity," IEEE Photon. Technol. Lett., vol. 23, no. 20, pp. 1520-1522, Oct. 2011

[23] T. Han, Y.-g. Liu, Z. Wang, J. Guo, Z. Wu, M. Luo, S. Li, J. Wang, and W. Wang, "Control and design of fiber birefringence characteristics based on selective-filled hybrid photonic crystal fibers," Opt. Express, vol. 22, no. 12, pp. 15002-15016, Jun. 11, 2014.

[24] Y. Liu, Y. Wang, B. Sun, C. Liao, J. Song, K. Yang, G. Wang, Q. Wang, G. Yin, and J. Zhou, "Compact tunable multibandpass filters based on liquid-filled photonic crystal fibers," Opt. Lett., vol. 39, no. 7, pp. 2148-2151, Apr. 1, 2014. 